\title{
Uso de Canna glauca para el tratamiento de aguas residuales domésticas en un humedal artificial de flujo superficial
}

\section{Use of Canna glauca for domestic wastewater treatment in anartificial surface flow wetland}

RODRIGUEZ-MARTINEZ, Nellybeth†**, SÁNCHEZ-HERRERA, Susana Graciela, NOGUEZESTRADA, Juan y PONCE-LIRA, Brenda

Universidad Politécnica de Francisco I Madero

ID $1^{\mathrm{er}}$ Autor: Nellybeth, Rodríguez - Martínez / ORC ID: 0000- 0001-7805-5958, Researcher ID Thomson: X-2132-2018, CV CONACYT ID: 96541

ID $1^{\text {er }}$ Coautor: Susana Graciela, Sánchez-Herrera / ORC ID: 0000-0002-7092-4693, CVU CONACYT ID: 37275

ID $2^{\text {do }}$ Coautor: Brenda, Ponce-Lira / ORC ID: 0000-0002-4326-6242, CVU CONACYT ID: 69895

ID $3^{\text {er }}$ Coautor: Juan, Noguez-Estrada / ORC ID: 0000 - 0002-0493-8843, Researcher ID Thomson: X- 4052 - 2018

\section{Resumen}

Se llevó a cabo el diseño y construcción de un prototipo de un humedal artificial de flujo superficial, con el objetivo de depurar agua residual doméstica. Se realizó la toma datos de campo, se diseñó y construyó el reactor y se colocó platanillo (Canna glauca) para evaluar la eficiencia de la planta en la depuración de aguas residuales. Para el diseño se consideró la ubicación, superficie disponible y se contabilizo el flujo del influente, la retención hidráulica, la dimensión del lecho rocoso, el volumen del tanque de almacenamiento. Se consideró la resistencia (mecánica, presencia de sulfatos y sustancia de limpieza y sanitización) y permeabilidad, para garantizar su vida útil. Se construyó un prototipo donde se caracterizó la calidad del agua por medio de una sonda multiparamétrica HI 9828-0®, en el afluente y efluente del reactor. De los resultados más relevantes se tiene que, la cantidad de oxígeno, el pH, la $\mathrm{CE}$, la resistividad, los STD, salinidad y el ORP, son parámetros que se vieron favorecidos con la presencia de la planta acuática para la depuración del Agua Residual.

Humedal, Agua Residual, Tratamiento de Agua

\begin{abstract}
The design and construction of a prototype of an artifi cial surface flow wetland was carried out with the aim of purifying domestic waste water. Field data was taken the reactor was designed and built to assess the plant's (Canna glauca) efficiency in sewage treatment. The location, available surface, and the influent flow were considered for the design; as well as available surface and the flow of influence of the influent the hydraulic retention, the size of the rock bed, the volume of the storage tank, resistance (mechanical, presence of sulfates and cleaning and sanitization substance) and permeability were considered to ensure its service life. A prototype was built where water quality was characterized by a HI 9828-0® multiparametric probe, in the reactor tributary ans effluent. Within the most relevant results is that the amount of oxygen, $\mathrm{pH}, \mathrm{EC}$, resistivity, STDs, salinity and ORP, are parameters that were favored with the presence of the aquatic plant for the purification of waste water
\end{abstract}

Wetland, WasteWater, Water Treatment

Citación: RODRIGUEZ-MARTINEZ, Nellybeth, SÁNCHEZ-HERRERA, Susana Graciela, NOGUEZ-ESTRADA, Juan y PONCE-LIRA, Brenda. Uso de Canna glauca para el tratamiento de aguas residuales domésticas en un humedal artificial de flujo superficial. Revista de Energía Química y Física. 2019. 6-18: 53-60

\footnotetext{
* Correspondencia al Autor (Correo electrónico: nrodriguez@upfim.edu.mx)

$\dagger$ Investigador contribuyendo como primer Autor
} 


\section{Introducción}

El crecimiento demográfico, la urbanización, la industrialización y el aumento de la producción y el consumo, han generado una demanda de agua dulce cada vez mayor (WWDR, 2018), se prevé que en 2030 el mundo tendrá que enfrentarse a un déficit mundial del $40 \%$ de agua, bajo un escenario climático donde el comportamiento actual se mantenga (UNESCO, 2015).

Para el año 2050, debido al crecimiento de la población mundial, se prevé que la demanda de agua aumente en casi un tercio (WWDR, 2018) lo que represnta que la demanda hidrica haya aumentado un 55\%, debido principalmente a demandas relacionadas con la creciente urbanización en los países en desarrollo (OECD, 2012a).

Las estimaciones recientes sugieren que el cambio climático será responsable de alrededor del $20 \%$ del incremento de la escasez global de agua (UNESCO, 2015). El acceso al agua y al saneamiento está reconocido como un derecho humano, y ha sido durante mucho tiempo el foco de políticas y objetivos de desarrollo internacional (UNCESCR, 2003; UNGA, 2010).

En muchos países se ha aumentado el saneamiento construyendo redes de alcantarillado, pero sin prestarle la debida atención al tratamiento y eliminación de las aguas residuales. Incluso en los países con unas rentas medio-altas, las aguas residuales de aproximadamente el $75 \%$ de los hogares con conexión a la red de alcantarillado pueden no recibir el tratamiento adecuado (Baum et al., 2013).

Se prevé que el cambio climático tendrá efectos directos sobre los organismos individuales, sobre las poblaciones y sobre los ecosistemas. En cuanto a los individuos, se ha encontrado que el cambio climático podría afectar su desarrollo, fisiología y sus comportamientos durante las fases de crecimiento, reproducción y migración. Por otra parte, es probable también que la modificación en los patrones de precipitación y el aumento de la temperatura (Böhning-Gaese, Jetz, \& Schaefer, 2008) afecten la distribución, tamaño, estructura y abundancia de las poblaciones de algunas especies.
Lo anterior, sumado a los efectos del cambio climático sobre el ciclo hidrológico, podría afectar las interacciones entre las especies, los ciclos de nutrientes y el funcionamiento, estructura y distribución misma de los ecosistemas. Esto, traería como consecuencia la alteración en los flujos y calidad de los servicios ambientales que prestan los ecosistemas (IPCC, 2007).

\section{Justificación}

Estudios dedicados a evaluar la disponibilidad de recursos en diferentes áreas del planeta auguran que los problemas asociados con la escasez de agua, la sequía y la contaminación de los recursos hídricos cobrarán mayor relevancia en las próximas décadas. (T. Buerkle, 2007). Estos problemas surgirán, según algunas fuentes, incluso en regiones que actualmente se consideran ricas, en lo que se refiere a recursos hídricos (PNUD, 2006). Si a esto añadimos que, actualmente, 1.2 billones de personas no tienen acceso a agua potable, 2.6 billones no tienen acceso a redes de saneamiento y millones de personas mueren anualmente, entre ellas 3900 niños al día, debido a enfermedades contraídas por el consumo de agua contaminada o a la falta de redes de saneamiento, la búsqueda de soluciones se convierte en un reto de una escala considerable. (M.A. Montgomery, 2007).

Diferentes actuaciones y tratamientos están siendo desarrollados con el fin de mejorar tanto la disponibilidad de recursos hídricos como la calidad de de los mismos. Entre ellas, podemos incluir la implementación de herramientas de gestión como los analisis de agua virtual (J.A. Allan, 1998) o huella hídrica (A.Y. Hoekstra, 2010); las políticas incentivadoras dedicadas a promover el uso responsable de los recursos (M. Qureshi, et al., 2010) o la búsqueda y desarrollo de tecnologías de tratamiento de agua donde se ahonde en la sostenibilidad y en la eficiencia energética (M.A. Shannon, et al, 2008).

En estos últimos años, este último aspecto ha alcanzado una gran relevancia dentro de la comunidad científica, desarrollándose numerosos estudios englobados dentro de lo que se ha denominado el "Nexo Agua-Energía" (J.L. Schnoor, 2011). 


\section{Objetivos}

\section{Objetivo General}

Evaluar el efecto del platanillo (Canna glauca) sobre las las características físico-químicas del agua provenientes de un humedal artificial de flujo superficial para el tratamiento de aguas residuales domésticas.

\section{Objetivos específicos}

Caracterizar la eficiencia de Canna glauca en la remoción de nutrientes en un humedal artificial.

\section{Marco Teórico}

Las aguas residuales son las aguas usadas y los sólidos que por uno u otro medio se introducen en las cloacas y son transportadas mediante el sistema de alcantarillado. En general, las aguas residuales consisten de dos componentes: un efluente líquido y un constituyente sólido, conocido como lodo (Sorrequieta, 2004).

\section{Contaminantes tipo de las aguas residuals}

Según Jiménez en 2002, dentro de los principales contaminantes que se presentan en el agua residual, dependiendo de su origen se encuentran: Microorganismos patógenos, Desechos orgánicos, Sustancias químicas inorgánicas, Compuestos orgánicos, Sedimentos y materiales suspendidos, Sustancias radioactivas y calor.

Un humedal artificial es un nombre genérico para designar al hábitat de aguas abiertas y al de los terrenos inundados de manera permanente o semipermanente. En este crecen y se desarrollan diferentes tipos de vegetales, animales y microorganismos adaptados a estas condiciones de inundaciones temporales y/o permanentes. En este tipo de ecosistema se desarrollan también determinados procesos físicos y químicos capaces de depurar el agua ya que eliminan grandes cantidades de materia orgánica, sólidos en suspensión e incluso productos tóxicos. (Gallego, 2011).

Un humedal artificial de flujo superficial proporciona tratamiento de aguas residuales a través de la floculación y sedimentación durante el flujo de aguas residuales a través de plantas acuáticas que crecen de pie en aguas poco profundas.
En algunos también hay áreas abiertas donde la bioxidación aeróbica complementa los procesos de eliminación física. Estos sistemas se asemejan a los humedales naturales en la función y la apariencia. (Jumbo, 2012)

Los dos mecanismos principales en el trabajo en la mayoría de los sistemas de tratamiento son las separaciones líquido/sólido y las transformaciones constituyentes. Las separaciones suelen incluir la separación por gravedad, filtración, absorción, adsorción, intercambio iónico, extracción y lixiviación. Las transformaciones pueden ser químicas, incluyendo las reacciones de oxidación / reducción, floculación, reacciones ácido / base, precipitación, o una serie de reacciones bioquímicas que ocurre en condiciones aeróbicas, anóxicas oanaeróbicas (Pereíra, 2011).

Los componentes principales de un humedal artificial tipo están constituidos por: El agua es la fase móvil dentro del humedal, la encargada del transporte de los contaminantes y en la cual se van a producir la mayoría de las reacciones responsables de la depuración.

El relleno, junto con los sedimentos y los restos de vegetación, son importantes por varias razones:

- Actúan como barrera primaria de tamizado.

- Actúan como estructura soporte de las plantas y como superficie para el crecimiento y desarrollo de la masa microbiana.

- Facilitan los mecanismos de adsorción e intercambio iónico entre el agua residual y los componentes minerales del suelo.

- La permeabilidad del relleno afecta al movimiento del agua a través del humedal.

Favorecen la precipitación química de contaminantes disueltos.

El sedimento orgánico es un elemento fundamental en el proceso de depuración por humedales, ya que sirve de sustrato para el crecimiento de multitud de microorganismos, presenta una elevada capacidad de cambio que asegura la retención y posterior transformación del material orgánico e inorgánico. 
La actividad microbiana, transforma un gran número de sustancias orgánicas e inorgánicas en sustancias inocuas o insolubles. Altera las condiciones de las reacciones de reducción-oxidación, del substrato y así afecta la capacidad de proceso del humedal. Está involucrada en el reciclaje de nutrientes. Algunas transformaciones microbianas son aerobias (es decir, requieren oxígeno libre) mientras otras son anaerobias (tienen lugar en ausencia de oxígeno libre). Muchas especies bacterianas son facultativas, es decir, son capaces de funcionar bajo condiciones aerobias $y$ anaerobias en respuesta a los cambios en las condiciones medioambientales.

Las poblaciones microbianas se ajustan a los cambios en el agua que les llega y se pueden extender rápidamente cuando se tiene la suficiente energía. Cuando las condiciones medioambientales no son convenientes, muchos microorganismos se inactivan y pueden permanecer inactivos durante años. La comunidad microbiana de un humedal construido puede ser afectada por sustancias tóxicas, como pesticidas y metales pesados, y debe tenerse cuidado para prevenir que tales sustancias se introduzcan en las cadenas tróficas en concentraciones perjudiciales.

Substrato: Los suelos con componentes húmicos y de arena altos son más fáciles para los tubérculos y los corredores para migrar a través y la colonización vegetal y el crecimiento es más rápido.

Realce de la estética y paisaje: Aunque los humedales son principalmente sistemas de tratamiento, proporcionan beneficios intangibles a la estética del sitio y mejoran el paisaje. Visualmente, los humedales son ambientes extraordinariamente ricos. Introduciendo el elemento agua, el humedal construido, tanto como el natural, agrega diversidad al paisaje. Pueden construirse humedales artificiales siguiendo las formas que tienen los contornos naturales del sitio, hasta el punto de que algunos humedales, para el tratamiento de agua son indistinguibles a simple vista.

Revestimiento: El requisito de revestimientos en los humedales artificiales depende de los requisitos reglamentarios de cada lugar y/o las características de la superficie y el subsuelo (Morales P. A., 2014)

\author{
Parámetros físicos-químicos y biológicos \\ relacionados con la depuración de aguas \\ contaminadas
}

\section{Oxígeno disuelto}

El oxígeno disuelto, es el factor que indica la potencialidad de respiración de los organismos acuáticos. Su disminución (ausencia de oxígeno) promueve procesos anaerobios, anulándose la probabilidad de actividades de fauna ictícola y otros seres acuáticos. Cualquier actividad de organismos aeróbicos, requiere un mínimo de oxígeno en los cuerpos de agua, estableciéndose límites no inferiores a $2 \mathrm{mg}^{-1}$ de oxígeno. Valores aceptables son considerados los superiores a $6 \mathrm{mg} \mathrm{l}^{-1}$. (Rodier, 2011).

\section{Resistividad}

La unidad estándar de resistencia eléctrica es el ohm y la resistividad de las aguas se expresa convenientemente en megaohms-centímetro. La conductividad se expresa en el valor recíproco, normalmente como microsiemens por centímetro $\left(\mu \mathrm{S} \mathrm{cm}^{-1}\right)$. Para el agua ultrapura los valores respectivos son de 18,24 Mohms.cm y $0,05483 \mu \mathrm{s} / \mathrm{cm}$ a $25^{\circ} \mathrm{C}$. (Moreno, 2011)

\section{El potencial Hidrógeno}

Es una medida que indica la acidez del agua. El rango varía de 0 a 14, siendo 7 el rango promedio (rango neutral). Un $\mathrm{pH}$ menor a 7 indica acidez, mientras que un $\mathrm{pH}$ mayor a 7 , indica un rango básico. Por definición, el $\mathrm{pH}$ es en realidad una medición de la cantidad relativa de iones de hidrógeno e hidróxido en el agua. Agua que contenga más iones de hidrógeno tiene una acidez mayor, mientras que agua que contiene más iones de hidróxido indica un rango básico (Rodier, 2011).

\section{Conductividad}

Es la medición de la habilidad del agua para transportar corriente eléctrica. Depende en gran medida en la cantidad de materia sólida disuelta en el agua (como la sal), teniendo rangos aceptables mínimos de $0.00 \mathrm{MS} \mathrm{cm}^{-1}$ a máximos de 2000.00 .C.E. $=$ Conductividad eléctrica a 25 ${ }^{\circ} \mathrm{C}$, en dS m${ }^{-1}$ (Moreno, 2011). 


\section{Temperatura}

Existe un intervalo de temperatura de 15 a $30{ }^{\circ} \mathrm{C}$, en el cual los microorganismos trabajan mejor si la temperatura del agua permanece alrededor de $20^{\circ} \mathrm{C}$. Cada aumento de $10^{\circ} \mathrm{C}$ en la temperatura duplica la velocidad de reacción. Sin embargo, cuando la temperatura del agua alcanza los 35 ${ }^{\circ} \mathrm{C}$, la velocidad de crecimiento decae rápidamente. Cuando la temperatura disminuye empieza a ser más lenta la velocidad de reacción. (Violeta E. Escalante Estrada, 2006), este factor debe ser monitoreado debido al aporte de agua caliente procedente del aseo y las tareas domésticas, consrvando una temperatura que oscile entre $10^{\circ} \mathrm{C}$ y $21^{\circ} \mathrm{C}$, con un valor medio de $15^{\circ} \mathrm{C}$, aproximadamente. (García, 2012)

\section{Sólidos disueltos totals}

El término sólido hace alusión a materia suspendida o disuelta en un medio acuoso. La determinación de sólidos disueltos totales mide específicamente el total de residuos sólidos filtrables (sales y residuos orgánicos) a través de una membrana con poros de $2.0 \mu \mathrm{m}$ (o más pequeños). Los sólidos disueltos afectan la calidad de un cuerpo de agua o un efluente de varias formas (Pérez \& Rodríguez, 2008).

\section{La salinidad}

Es una propiedad importante de aguas usadas y de cuerpos de agua naturales. Este parámetro representa una medida de la cantidad total de sales disueltas en un volumen determinado de agua. El contenido de sales suele ser peligroso cuando es superior a $100 \mathrm{mg} \mathrm{l}^{-1}$, contabilizándose en esta cifra todos los iones existentes en el agua. (Moran, 2014)

\section{Potencial de Oxido Reducción}

La cuantificación del potencial de óxido reducción es la que se establece entre un electrodo inerte (platino o de oro), y una disolución de las formas oxidada y reducida de un ión. Cualquier sistema o ambiente que acepte electrones de un electrodo normal de hidrógeno es una media celda con un potencial redox positivo. (Moran, 2014)

Un valor oxido reducción positivo y de alta magnitud es indicativo de un ambiente que favorece las reacciones de oxidación.
Del otro lado, un valor negativo y de baja magnitud es indicativo de un ambiente altamente reductor. Los microorganismos anaerobios facultativos demuestran actividad metabólica sobre un rango amplio de valores oxido reducción. Estos utilizan oxígeno como aceptor final de los electrones a valores altos. Cuando el potencial redox es bajo, algunos de estos microorganismos llevan a cabo reacciones de fermentación mientras otros obtienen energía a través de la respiración anaerobia. (Poole, 1979).

\section{Selección de Vegetación}

Según García S.J. y Corzo, H.A. en 2008, la selección de la vegetación usada en un sistema de humedales debe tener en cuenta las características de la región donde se realizará el proyecto, así como las siguientes recomendaciones:

1. Las especies deben ser colonizadoras activas, con eficaz extensión del sistema de rizomas, además de alcanzar una biomasa considerable para una mayor asimilación de nutrientes para potenciar el crecimiento de la biopelícula.

2. Deben disponer de un sistema eficaz de transporte de oxígeno hacia las partes subterráneas para promover la degradación aeróbica y la nitrificación.

3. Las especies utilizadas deben estar adaptadas a las condiciones ambientales del sitio.

4. Las especies deben tolerar los contaminantes presentes en las aguas residuales.

5. Se deben utilizar especies propias de la flora local.

El uso de macrófitas flotantes ha permitido eficiencias de remoción significativamente altas en todos los constituyentes de las aguas residuales. Una cantidad importante de especies ha sido empleada en sistemas de tratamiento, y en ejercicios investigativos a escala real y a escala laboratorio, siendo el jacinto de agua, la macrófita de mayor interés. Esta especie alcanza reducciones de $\mathrm{DBO}_{5}$ en el orden de $95 \%$ y hasta 90,2\% para la DQO. En el caso de los sólidos suspendidos se registran disminuciones con valores que se encuentran en el rango de $21 \%$ y 91\%. (Martelo, 2012). 
Otra planta utilizada para la depuración del agua, es el Alcatraz (Zantedeschia aethiopica), originaria de zonas templadas, su evaluación en humedales artificiales, en México reporta beneficios adicionales por su valor económico; sin embargo, es susceptible a heladas y a algunas plagas y enfermedades, esta especie incrementa la efectividad en la remoción de la DQO en $13 \%$, la $\mathrm{DBO}_{5}$ en $13 \%$, los fosfatos como $\mathrm{PO}_{4}$ en $17.67 \%$, N-amoniacal en $10.6 \%$, SST en $9.62 \%$, conductividad eléctrica en $5.3 \%$, turbiedad en $11.02 \%$, sulfatos como SO4 en $9.25 \%$, coliformes totales en $1.21 \%$ y coliformes fecales en $1.46 \%$, con respecto a un pantano sin plantas emergentes (Avelar, 2011)

\section{Características de la Canna glauca}

Se refiere a una especie nativa de gran parte de América, desde los Estados Unidos hasta el centro de Argentina, crece desde el noroeste hasta el norte, en lugares húmedos y bajos aunque no es una especie muy frecuente, es más común de observar en terrenos modificados cerca de poblados y caminos. Mide entre 1 y 2 metros de altura. Posee una inflorescencia terminal en forma de ramo que llega a comprender 10 flores, de pétalos amarillos o de otros colores (Fig. 1). El fruto es una cápsula elíptica de entre 2 y $5 \mathrm{~cm}$. La polinización es llevada a cabo por aves (Zingiberales, 2011).

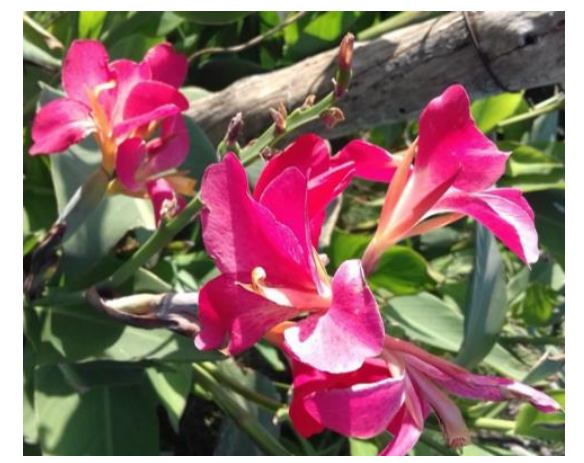

Figura 1 Floración de la planta Canna glauca (Platanillo

\section{Metodología}

La presente investigación se llevó a cabo en la Universidad Politécnica de Francisco I. Madero, estado de Hidalgo, México. Para evaluar el efecto de la Canna glauca sobre la calidad del agua. Se realizó la caracterización del efluente e influente utilizando una sonda multiparamétrica HANNA Instruments HI $9828-0^{\circledR}$ para calidad del agua, sumergiendo los electrodos y registrando parámetros como:
Oxigeno disuelto (OD), Conductividad Eléctrica (CE), pH, Temperatura (T) y Sólidos totales disueltos (STD) a la entrada y a la salida del humedad. Dichos parámetros se tomaron semanalmente por un lapso de 10 semanas consecutivas.

\section{Resultados}

De los datos obtenidos se obtuvo que la concentración de oxigeno disuelto a la salidad del humedal, fue altamente significativo, incrementando en hasta $4.3 \mathrm{mg} \mathrm{l}^{-1}$ de OD con respecto a la entrada $\left(0.36 \mathrm{mg} \mathrm{l}^{-1}\right)$ lo cual, indica la efectividad del tratamiento con el uso de Canna, esta condición de OD incrementa la probabilidad de que se promuevan procesos anaerobios que favorezcan actividades de fauna ictícola y otros seres acuáticos, según lo refiere Rodier en 2011 (Figura 2).

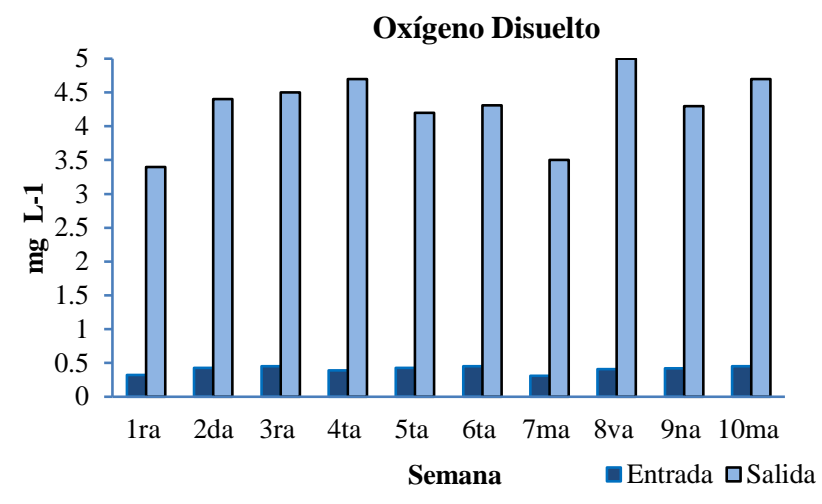

Figura 2 Concentración de oxigeno disuelto en entrada y salida del humedal

En Relación a la conductividad eléctrica, la figura 3 , muestra que los valores promedio a la entrada del reactor $\left(2.242 \mathrm{dS} \mathrm{m}^{-1}\right)$, fueron mayores que los registrados a los promedios generados a la salida (1.492 dS m-1), esto debido al proceso de depuración del tren de tratamiento, el cual permite que el valor disminuya $0.32 \mathrm{dS}$ $\mathrm{m}-1$. Lo anterior, corrobora la efectividad de la filtración del humedal y de la Canna glauca.

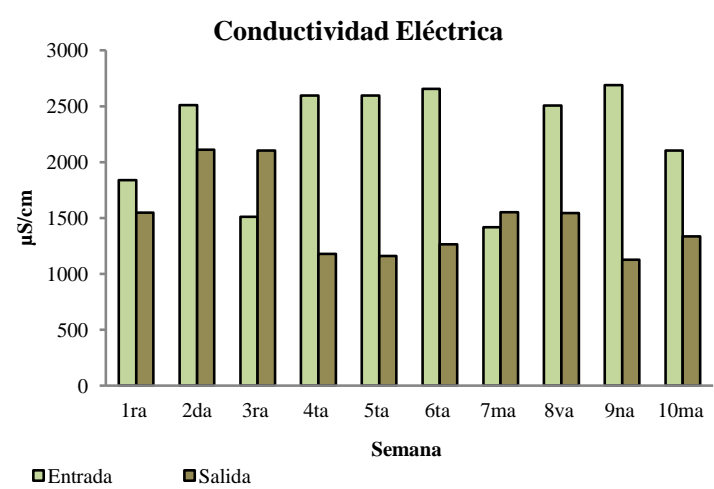

Figura 3 Lecturas de Conductividad Eléctrica en el reactor 
La figura 4, muestra la eficiencia del humedal en la fluctuación del $\mathrm{pH}$, cuyos valores a la entrada (alcalino, 9.01 en promedio,) tienden a decrecer de manera paulatina durante la toma de datos. Una vez que pasa el agua por el humedal se registró un promedio de $\mathrm{pH}$ de 7.53 , lo cual clasifica al valor como neutro. Este efecto es deseable debido a que la mayoría de los cultivos se desarrolla en este rango de $\mathrm{pH}$, razón por la cual es agua apta para la producción de cultivos (Figura 4).

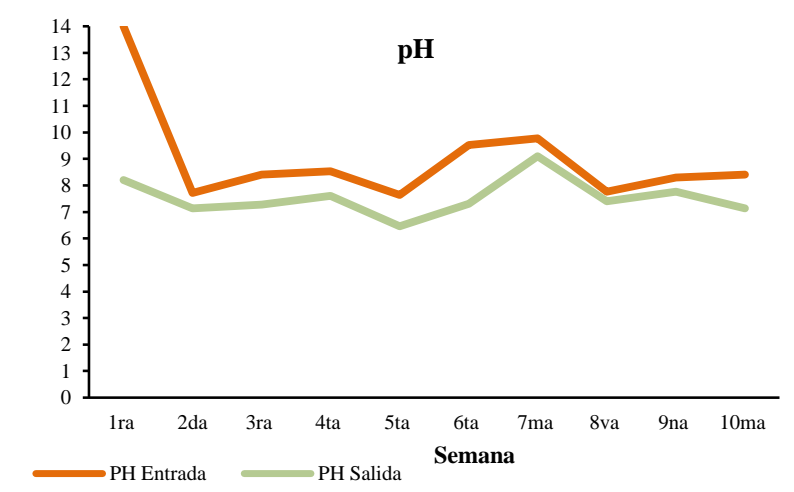

Figura 4 Comportamiento del potencial hidrógeno en humedal

La temperatura es un parametro importante debido a que se puede minimizar la velocidad de reacción de los organismos presentes en el reactor, en este caso, los valores se situaron en el rango de 14.5 a $18.1{ }^{\circ} \mathrm{C}$, en la entrada y de 16.5 y $12.9^{\circ} \mathrm{C}$ a la salida (Figura $5)$.

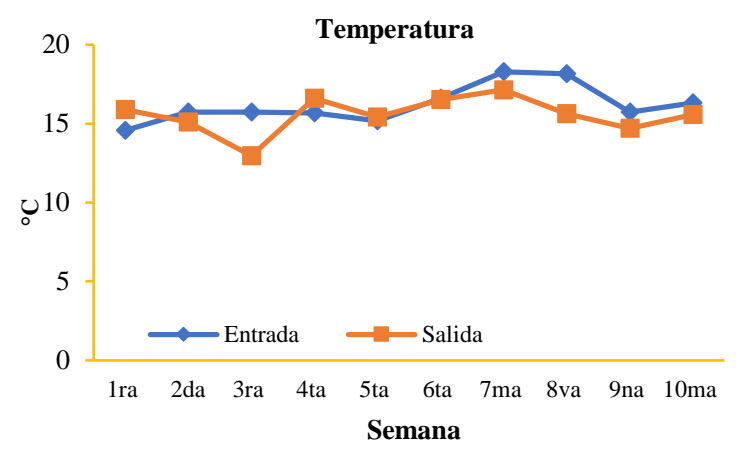

Figura 5 Temperatura presente en el humedal

En relación a sólidos totales disueltos evaluados a la entrada del prototipo, se registró un valor promedio de $1224.5 \mathrm{ppm}$. Los valores decrecieron considerablemente a partir de la cuarta semana de monitoreo, donde se alcanzó un promedio de $55 \%$ de remoción a la salida.
El comportamiento de este parametro se ve modificado con la agregación del agua residual en el prototipo aparentemente en una relación directamente proporcional, esto indica la eliminación de particulas presentes en el agua debido a la presencia de Canna glauca.

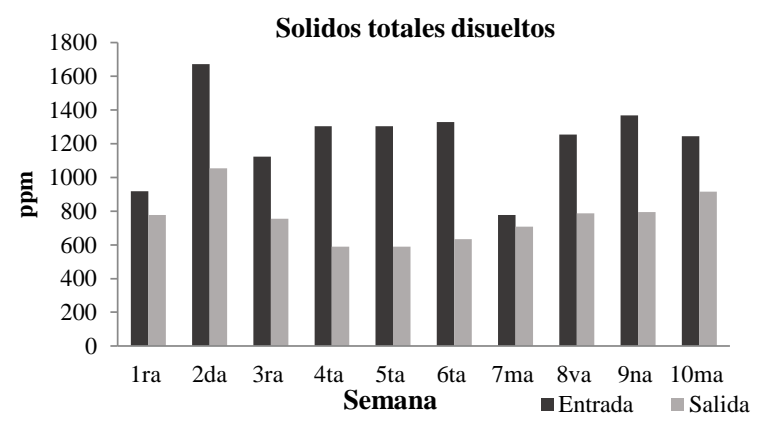

Figura 5 STD presentes a la entrada y salida del humedal

\section{Conclusiones}

El uso de un humedal artificial permite el cumplimiento a los reglamentos de zonificación y construcción establecido como proporción de áreas verdes en casas habitación, favorecen el paisajismo, otorga beneficios al ecosistema al tratar las aguas residuales domiciliarias, evitando el vertido de agua contaminada a los causes de agua.

El uso de este tipo de obras civiles y la utilización de especies depuradoras como la Canna glauca, proporciona el mejoramiento de la calidad del agua doméstica, permitiendo la reutilización del agua residual en los domicilios y garantiza la sanidad de las personas

\section{Referencias}

Arias., F. G. (1999). El Proyecto de Investigación: Guía . Obtenido de http://www.smo.edu.mx/:

http://www.smo.edu.mx/colegiados/apoyos/pro yecto-investigacion.pdf

Blanca Elena Jimenez Cisneros. XXVIII Congreso Interameticano de Ingeniería Sanitaria y Ambiental. Cancún. México Riego agrícola con agua residual y sus impliacciones en la salud. Caso práctico. 2002.

Böhning-Gaese, K., Jetz, W., \& Schaefer, H.-C. (2008), Impact of climate change on migratory birds: community reassembly versus. Global Ecology and Biogeography, 38-49. 
Buerkle, T. 2007. Making every drop count, in, Food and Agriculture Organization of the United Nations.

Gallego A. I. Y. 2011. Monografia sobre humedales artificial de flujo subsuperficial para remoción de metales pesados en aguas residuales. KinectMath: http:// KinectMatch.org/.

García S.J. y Corzo, H. A., 2008. Depuración con humedales construidos. Guía parctica de deseño, Construcción y explotación de sistemas de humedales de flujo subsuperficial. Departamento de Ingenieria Hidraulica, Maritima y Ambiental.

Hoektra, A. Y., 2010. The relation between international trade and freshwater scarcity. Working paper ERSD-2010-05 World Trade.

Informe de las Naciones Unidas sobre los Recursos Hídricos en el Mundo 2015. Disponoible en http://www.unesco.org/new/fileadmin/MULTI MEDIA/HQ/SC/images/WWDR2015Facts_Fig ures_SPA_web.pdf

IPCC (2013a), Cambio Climático 2013-Bases Físicas. Resumen para Responsables de Política. Estados Unidos de América.

J.A. Allan .1998. Virtual Water: A strategic resourse global solutions to regional deficits, Ground Water, 36.545-546

J.L. Schnoor, Water-Energy Nexus, Environmental science \& technology, 45 (2011) 5065-5065.

Luh J, Baum R, Bartram J. Equity in water and sanitation: Developing an index to measure progressive realization of the human right. Int $\mathrm{J}$ Hyg Environ Health 2013; 216(6):662-671.

M.A. Montgomery, M. Elimelech. 2007. Water and sanititation in developing countries: Including health in the equiation, Enviromental Science and tecnology. 41, 17-24.

M.A. Shannon, P.W. Bohn, M. Elimelech, J.C. Georgiadis, B.J. Marinas, A.M. Mayes. 2008. Science and tecnology for water purification in the comming decades, Nature, 452,301-310.
Pérez, A. \& Rodriguez, A.. 2008. Indicie fisicoquimico de la calidad de agua para el manejo de lagunas tropicales de inundación. Rev. Biol. Trop. Vol. 56 Num. 4 pp. 1905 1918.

PNUD, 2006. Beyond scarcity: Power, poverty and the global water crisis in Naciones Unidas.

Qureshi, M. K. Schwabe, J. Connor, M. Kirby. 2010. Enviromental water incentive policy and return flows, Water Resources Reseaech. Pp. 46 Sitios web

Sorrequieta, 2004. Aguas Residuales: reuso y tratamiento. Lagunas de estabilización: una opción para latinoamerica. Departamento de bioquimica Clínica. Tecnologia en salud publica de la Universidad Politécnica de Catalunya. Vol 1, pp. 16-18

UNESCO (2015) "El Crecimiento Insostenible Y La Creciente Demanda Mundial De Agua", Wwdr, p. 12. Disponible en: http://www.unesco.org/new/fileadmin/MULTI MEDIA/HQ/SC/images/WWDR2015Facts_Fig ures_SPA_we b.pdf

United Nations Committee on Economic, Social and Cultural Rights (UNCESCR). General Comment No. 15: The Right to Water (Arts. 11 and 12 of the Covenant). Geneva: UNCESCR; 2003. UN Document E/C.12/2002/11

WWDR, 2018. Informe Mundial de las Naciones Unidas sobre el Desarrollo de los Recursos Hídricos 2018: Soluciones basadas en la naturaleza para la gestión del agua., Paris: s.n 